\title{
Methylprednisolone-Responsive Leptospiral Acute Pulmonary Syndrome: A Case Report
}

\author{
Iman D. Johan-Arief,' Shen H. Lee, ${ }^{1}$ Xin Y. Er,' Ganesh Kasinathan, ${ }^{2}$ Naganathan Pillai., ${ }^{1,2}$
}

\begin{abstract}
Background: Leptospirosis is an infectious disease caused by the spirochete of the genus leptospira. It is thought to be the most common zoonosis globally and has a wide range of clinical presentations with pulmonary hemorrhage being one of its most severe manifestations. This entity known as acute pulmonary syndrome carries a high fatality rate. However, it can be effectively managed with methylprednisolone therapy. Case: We report a case of leptospirosis in a 26-year-old Bangladeshi male who was otherwise healthy. He presented with a 7-day history of fever with chills and rigors, and hemoptysis for a duration of 2 days. Physical examination revealed a febrile and lethargic man. Respiratory examination exhibited bilateral generalized crepitations over the lung fields. A chest radiograph performed showed bilateral alveolar shadowing. The diagnosis of leptospirosis was made based on positive Immunoglobulin M enzyme-linked immunosorbent assay serology, which was then confirmed by the microscopic agglutination test for leptospirosis. The patient was commenced on intravenous antibiotics and methylprednisolone at this time. He responded well clinically with resolution of fever and hemoptysis and a marked decrease in crepitations upon auscultation. This correlated with radiological improvement evidence by an obvious reduction in alveolar shadowing on subsequent chest radiograph 2 days later. Conclusion: This case is highly pertinent to the medical field as leptospirosis is an ever-growing problem and acute pulmonary syndrome is an emerging manifestation of it. Therefore, early recognition and intervention is required as this can be effectively treated with methylprednisolone therapy even in resource-limited settings.
\end{abstract}

Keywords: Fever; Hemoptysis; Leptospirosis; Methylprednisolone; Agglutination Tests (Source: MeSH, NLM).

About the Author: Iman Daniel is currently a final-year medical student at Monash University Malaysia. He has a keen interest in pathology and was offered the Pathology Update 2015 Conference scholarship to attend the conference in Melbourne, Australia.
Submission: Jul 14, 2015

Acceptance: Nov 23, 2015

Publication: Dec 4, 2015

Process: Peer-reviewed

${ }^{1}$ Monash University, Selangor, Malaysia.

${ }^{2}$ Segamat Hospital, Segamat, Malaysia.

\section{Correspondence:}

Iman Daniel Johan-Arief

Address: Jalan Lagoon Selatan, Bandar Sunway, 47500 Subang Jaya, Selangor, Malaysia

Email: STRY9@mail.umkc.edu

\section{Introduction}

Leptospirosis is a very common zoonotic infection, with a geographically ubiquitous distribution worldwide. It is caused by a thin and coiled spirochete under the genus of leptospira and 21 species of leptospira have been identified, 13 of which are considered pathogenic. ${ }^{1}$ Leptospirosis is an emerging infectious illness, with higher incidences in warm, humid, tropical countries.

Leptospiral acute pulmonary syndrome (APS) is an increasingly prevalent clinical entity that brings serious complications with a very high mortality rate. It is the most common cause of death in cases of leptospirosis. ${ }^{2}$ Symptoms include progressive hemoptysis and dyspnea which ultimately leads to patients dying from asphyxiation and respiratory failure. ${ }^{2}$ Thus, it is important to make a prompt diagnosis and initiate early treatment with the appropriate therapy. The microscopic agglutination test (MAT) is widely used in the confirmation of the diagnosis of leptospirosis.

Despite the notorious pulmonary manifestations of the disease, methylprednisolone therapy is highly effective in reducing morbidity and mortality rates. It is proposed that treatment within 12 hours of recognition of pulmonary involvement in leptospirosis can be lifesaving. ${ }^{3}$ In addition, methylprednisolone therapy also reduces the need for ventilator support and therefore, makes it a feasible choice when it comes to a hospital setting with limited resources. ${ }^{3}$ This case is unique, as there have only been a handful of similar cases within the literature which describes the efficacy of methylprednisolone in treating leptospiral acute pulmonary syndrome.

Key Points:
- Pulmonary hemorrhage is an emerging manifestation of leptospirosis,
with a high fatality rate if early intervention is not initiated.
- The pathophysiology of pulmonary hemorrhage in leptospirosis oc-
curs via an immune mediated pathway, with deposition of immunoglo-
bulins and complement on the pneumocytes, thus making immunosu-
ppressant and anti-inflammatory agents an excellent choice of therapy.
- Early intervention with high dose methylprednisolone for the first 3
days followed by oral prednisolone is effective in reducing the severity
of leptospiral acute pulmonary syndrome.
- Methylprednisolone therapy reduces the needs for tertiary referral
and critical care, thus enabling patients to be treated in a district set-
ting without further deterioration.

\section{The Case}

A 26-year-old male of Bangladeshi nationality, who was previously healthy, developed persistent fever of one-week duration. He presented to a district hospital in the state of Johor, Malaysia, with a high-grade fever that was associated with chills, rigors and body aches. On day 3 of the fever, he experienced a dry cough and shortness of breath, which then progressed to massive hemoptysis on day 5 . He denied any night sweats, rashes or lethargy. Systems review for cardiovascular, gastrointestinal, neurological and genitourinary systems were unremarkable. Past medical history and family history were not significant. He has no known allergies. There was no tuberculosis contact. He abstains from alcohol and denied any illicit drug use. He is a chronic smoker of 5 pack-years. He is married and has been working in Malaysia for a year. Currently, he is working in an oil palm plantation where his job involved constant contact with soil. He claimed to have gone swimming in a waterfall a week prior to the onset of fever. 


\section{Case Report}

Examination revealed a dehydrated patient with cool extremities. He had subconjunctival suffusion which is characterized by conjunctival redness especially on the palpebral conjunctiva. There was no obvious skin rash. Vital signs recorded a blood pressure of $101 / 82 \mathrm{mmHg}$. He was tachycardic at 118 beats per minute with a reduced radial pulse volume. His temperature was $38.20 \mathrm{C}$ and he had an oxygen saturation of $90 \%$ on room air. He was also jaundiced with an associated pallor. No cervical lymph nodes were palpable. Respiratory examination revealed bilateral generalized crepitations over the lung fields. Cardiovascular, abdominal and neurological examinations were unremarkable.

The complete blood count showed a mild anemia of $10.6 \mathrm{~g} /$ $\mathrm{dL}$, leukocytosis $(13.9 \times 109 / \mathrm{L})$ with predominant neutrophilia (absolute neutrophil count of $12.1 \times 109 / \mathrm{L}$ ) and significant thrombocytopenia $(48 \times 109 / \mathrm{L})$. His coagulation profile showed prolonged prothrombin time of 16.3 seconds and a normal activated partial thromboplastin time and international normalized ratio (INR). His renal profile showed urea of $7.1 \mathrm{mmol} / \mathrm{L}$, high serum creatinine (197 umol/L), hypokalemia (3.09 mmol/L), and a normal sodium level. The liver function tests showed deranged liver enzymes. The alanine transaminase (ALT) was $102 \mathrm{U} / \mathrm{L}$ and aspartate aminotransferase (AST) was $143 \mathrm{U} / \mathrm{L}$. The alkaline phosphatase (ALP) was $158 \mathrm{U} / \mathrm{L}$, albumin of $34 \mathrm{~g} / \mathrm{L}$ and total bilirubin of $85.2 \mathrm{umol} / \mathrm{L}$ with unconjugated bilirubin predominating. He also had an elevated C-reactive protein level of $15.8 \mathrm{mg} / \mathrm{L}$ and erythrocyte sedimentation rate of $97 \mathrm{~mm} / \mathrm{hr}$. Arterial blood gas exhibited features of type 1 respiratory failure and high anion gap metabolic acidosis with $\mathrm{pH}$ of 7.28, p02 $58 \mathrm{mmHg}$, normal $\mathrm{pCO}_{2}$ and bicarbonate of $17 \mathrm{mmol} / \mathrm{L}$. Urine examination was normal. Chest radiograph revealed bilateral alveolar shadowing as shown in Figure 1. 12-lead electrocardiogram and the 2-D transthoracic echocardiogram were normal.

Urine, sputum and blood samples for culture returned with no growth and no acid-fast bacilli were detected in the sputum samples. Blood film for malarial parasites did not reveal any evidence of parasitemia and serology for dengue was negative. Leptospirosis IgM ELISA was positive. A high titer of 1:400 microscopic agglutination test confirmed the diagnosis of leptospirosis.

With regards to this patient's management, he was started on intravenous tranexamic acid ( $500 \mathrm{mg}$ ) three times daily to stop the hemorrhage. He was immediately commenced on intravenous benzyl penicillin at a dose of $1200 \mathrm{mg}$ every 6 hours, which was maintained for 1 week. He was also given intravenous methylprednisolone $15 \mathrm{mg} / \mathrm{kg}$ daily for 3 days.

He responded well clinically to the treatment as shown by a marked reduction in hemoptysis. Pulmonary auscultation showed decreased crepitations bilaterally. Chest radiograph revealed a significant reduction in alveolar opacities on day 2 post-treatment as shown in Figure 2. He was discharged home on a tapering dose of oral prednisolone at $40 \mathrm{mg}$ for the first 2 days, $30 \mathrm{mg}$ for the subsequent 2 days and $20 \mathrm{mg}$ for the last 2 days. At follow-up one month later, he was symptom free and in good health. An informed written consent was obtained from the patient prior to the writing of this case report.
Figure 1. A posterior-anterior (PA) view of the patient's chest radiograph on admission showing gross alveolar shadowing, suggestive of intra-alveolar hemorrhage.

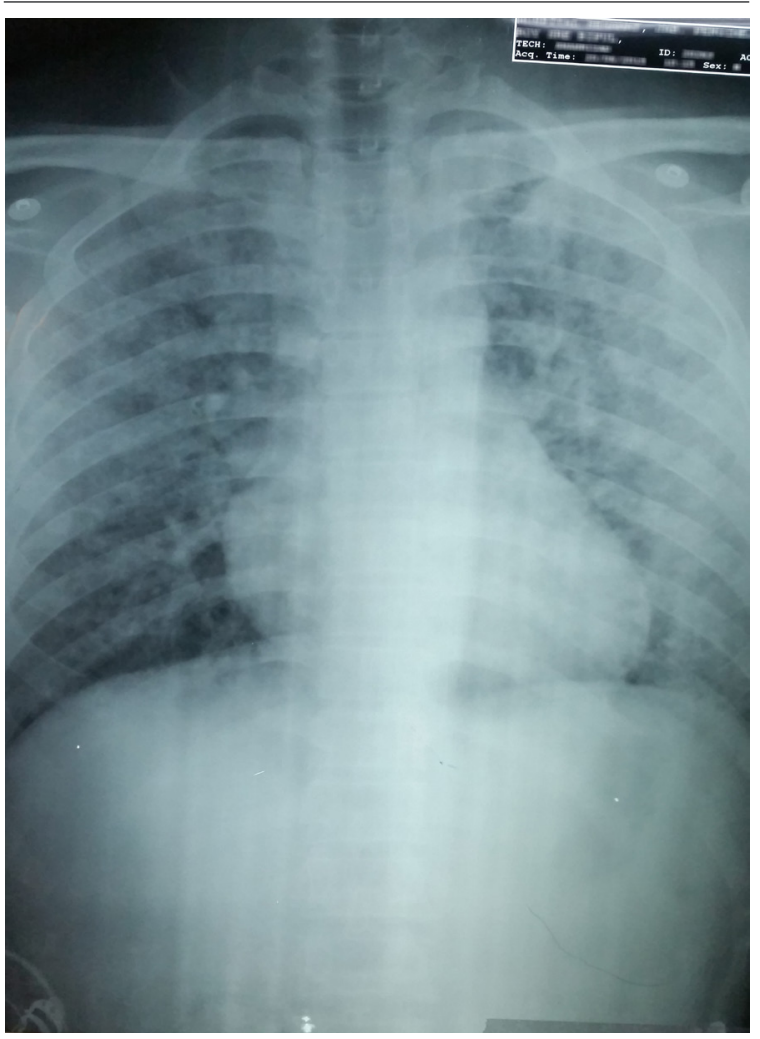

Figure 2. Patient's repeat chest X-ray (PA view) 2 days after IV methylprednisolone therapy, showing marked reduction in airspace nodular opacities and infiltration.

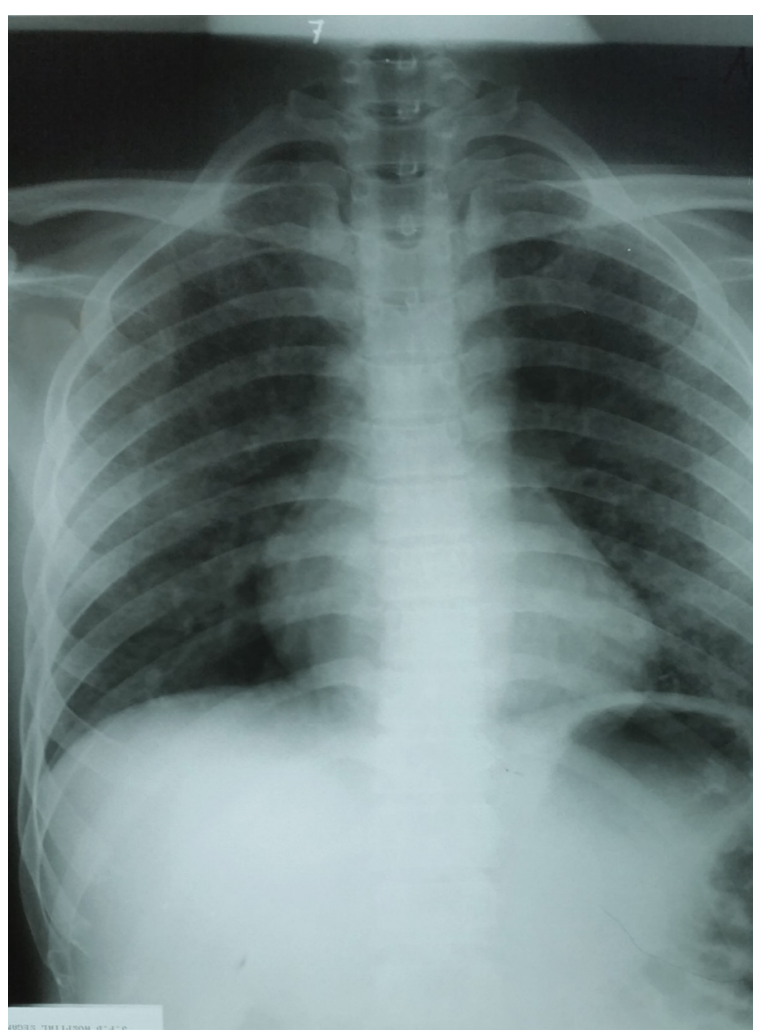




\section{Discussion}

Leptospirosis is an infectious illness, which is recently increasing in incidence. It is a medical problem with an annual incidence rate ranging from 10 to 100 cases per 100000 people annually in the tropical areas. Leptospirosis is usually transmitted through direct and indirect contact with animal urine. The incubation period for leptospirosis ranges from 2 to 30 days.' The presentation of this disease can be broadly classified into the following 4 groups: a mild viral flu-like illness, Weil's disease (acute kidney injury with hepatitis-like picture), meningitis or meningoencephalitis, and pulmonary hemorrhage with subsequent respiratory insufficiency. (World Health Organization. Available from: http://www.paho.org/hq/index. php?option=com_contentClview $=$ articlectid $=7821 \% 3$ Ainforma cion-general-leptospirosis- - $t$ catid $=4711 \% 3$ Aleptospirosis-homectltemid $=40695$ cllang $=$ en, updated 2015 January 15; cited 2015 Jul 11).

The gold standard investigation is the microscopic agglutination test (MAT), which involves the mixing of the patients' antibodies with live leptospira antigens. ${ }^{4}$ An agglutinate or a clump formation shows a positive reaction, which can then be viewed under dark field microscopy. ${ }^{4}$ One of the major drawbacks of this test is that it is highly dependent on the production of antibodies in the host. Thus, during the acute phase of the disease where the circulating leptospires have not evoked a full immune response, the antibody levels would be minimal and would subsequently lead to a false negative result. ${ }^{5}$

Leptospiral APS is manifested by profuse intra-alveolar hemorrhage with symptoms such as dyspnea, cough and hemoptysis developing on the 4th to 6th day of illness. ${ }^{2}$ This aggressive form of the disease has a fatality rate of $74 \%$ despite intensive and critical therapy being provided. ${ }^{6}$

The pathophysiology of leptospiral APS appears to be immune-mediated. ${ }^{4}$ The proposed mechanism begins with the activation of the endothelium which leads to an increase in vascular permeability which allows immunoglobulins such as IgG, IgM and IgA to leak out and deposit on the walls of type I and type II pneumocytes.? The activation and deposition of complement $C_{3}$ then ensues, resulting in cell necrosis and subsequent hemorrhage into the alveolar spaces resulting in a histopathological pattern that is not seen with other forms of pulmonary hemorrhage.? Leptospiral toxins also activate tumor necrosis factor alpha (TNF- $\alpha$ ), which is an acute phase reactant produced primarily by macrophages. ${ }^{8}$ Local effects of TNF- $\alpha$ triggers the cardinal signs of inflammation with systemic effects being fever and leukocytosis.

Multiple nodular densities which represent alveolar hemorrhage can be appreciated on the chest radiograph. These may progress to form a confluent consolidation that subsequently resolves, leaving an indistinct ground-glass pattern that is diffuse in nature. ${ }^{9}$
There are various corticosteroid therapies available in the rural setting such as hydrocortisone, dexamethasone, methylprednisolone and oral prednisolone. Intravenous hydrocortisone plays a huge role in acute exacerbations of asthma and chronic obstructive pulmonary disease (COPD). Oral prednisolone therapy is given as a short course following these exacerbations and also as a maintenance therapy for certain chronic cases. Intravenous dexamethasone on the other hand is indicated in cases of raised intracranial pressure. Intravenous methylprednisolone has been effectively used in cases of cerebral lupus and lupus nephritis. However, its availability in the rural setting is limited. Also, methylprednisolone with its high anti-inflammatory and immunosuppressive properties plays an important role in decreasing the severity of leptospiral APS. ${ }^{8}$ Pharmacologic doses of glucocorticoid-based therapy can reverse the effect of immune-mediated response and therefore, lessen the disease-associated vasculitis by reducing the cytokine and TNF- $\alpha$ production. ${ }^{8}$

In a study by Shenoy et al., $61.53 \%$ of patients showed significant improvement when treated with intravenous methylprednisolone $1 \mathrm{~g}$ daily for 3 days during the early course (within the first 12 hours) of pulmonary leptospirosis. ${ }^{3}$ It is also beneficial in severe cases where mortality was seen to decrease from $85.71 \%$ to $33.33 \% .^{3}$ The study also demonstrated a reduced need for ventilator support with methylprednisolone therapy. This data is useful for rural hospitals with limited resources.

In another study by Kularatne et al., it was observed that a regime of intravenous methylprednisolone 50omg for 3 days followed by oral methylprednisolone $8 \mathrm{mg}$ for 5 days played a significant role in reducing mortality rates due to pulmonary involvement specifically. ${ }^{10}$ This study also showed that the development of multiple organ dysfunction as the disease progressed can hinder the efficacy of methylprednisolone therapy.10 This is crucial since a delay in the hospitalization of patients with leptospiral APS in tropical areas due to an initial misdiagnosis of dengue fever has led to a poorer outcome of these patients. ${ }^{2}$

In conclusion, leptospiral APS carries a high mortality rate and is an emerging manifestation of leptospirosis. The success of intravenous methylprednisolone therapy as described in this case report shows that APS can be managed effectively in a district setting without any need for tertiary care in certain circumstances. 


\section{Case Report}

\section{References}

1. Ko Al, Goarant C, Picardeau M. Leptospira: the dawn of the molecular genetics era for an emerging zoonotic pathogen. Nat Rev Microbiol. 2009 $0 \mathrm{ct} ; 7(10): 736-47$.

2. Gulati S, Gulati A. Pulmonary manifestations of leptospirosis. Lung India. 2012 Oct;29(4):347-53.

3. Shenoy VV, Nagar VS, Chowdhury AA, Bhalgat PS, Juvale NI. Pulmonary leptospirosis: an excellent response to bolus methylprednisolone. Postgrad Med J. 2006 Sep;82(971):602-6.

4. Levett PN. Leptospirosis. Clin Microbiol Rev. 2001 Apr;14(2):296-326.

5. Natarajaseenivasan K, Raja V, Narayanan R. Rapid diagnosis of leptospirosis in patients with different clinical manifestations by $16 \mathrm{~S}$ rRNA gene based nested PCR. Saudi J Biol Sci. 2012 Apr;19(2):151-5.

6. Gouveia EL, Metcalfe J, de Carvalho AL, Aires TS, Villasboas-Bisneto JC, Queirroz A et al. Leptospirosis-associated severe pulmonary hemorrhagic syndrome, Salvador, Brazil. Emerg Infect Dis. 2008 Mar;14(3):505-8.
7. Croda J, Neto AN, Brasil RA, Pagliari C, Nicodemo AC, Duarte MI. Leptospirosis pulmonary haemorrhage syndrome is associated with linear deposition of immunoglobulin and complement on the alveolar surface. Clin Microbiol Infect. 2010 Jun; 16(6):593-9.

8. Thunga G, John J, Sam KG, Khera K, Khan S, Pandey S et al. Role of high-dose corticosteroid for the treatment of leptospirosis-induced pulmonary hemorrhage. J Clin Pharmacol. 2012 Jan;52(1):114-6.

9. Luks AM, Lakshminarayanan S, Hirschmann JV. Leptospirosis presenting as diffuse alveolar hemorrhage: case report and literature review. Chest. 2003 Feb;123(2):639-43.

10. Kularatne SA, Budagoda BD, de Alwis VK, Wickramasinghe WM, Bandara JM, Pathirage LP, et al. High efficacy of bolus methylprednisolone in severe leptospirosis: a descriptive study in Sri Lanka. Postgrad Med J. 2011 Jan;87(1023):13-7.

\section{Acknowledgments}

We would like to extend our appreciation to the Departments of Internal Medicine, Radiology and Microbiology and the director of Hospital Segamat for their kind assistance and motivation throughout our case study.

Conflict of Interest Statement a Funding

The author has no funding, financial relationships or conflicts of interest to disclose.

\section{Author Contributions}

Conception and design the work/idea, Administrative or technical advice: IDJA. Write the manuscript: IDJA, SHL, XYE. Critical revision of the manuscript: IDJA, XYEGK, NP. Approval of the final version: GK, NP. Contribution of patients or study material: SHL, XYE.

Cite as:

Johan-Arief ID, Lee SH, Er XY, Kasinathan G, Pillai N. Methylprednisolone-Responsive Leptospiral Acute Pulmonary Syndrome: A Case Report. Int J Med Students. 2015 Sep-Dec;3(3):159-62. 